\title{
Magnetisation and transport current loss of a BSCCO/Ag tape in an external $\mathrm{AC}$ magnetic field carrying an AC transport current
}

\author{
J. J. Rabbers, D. C. van der Laan, B. ten Haken and H. H. J. ten Kate \\ University of Twente, Faculty of Applied Physics, Low Temperature Division, P.O. Box 217, 7500 AE Enschede, The Netherlands
}

Abstract- In practical applications, BSCCO/Ag tapes are exposed to external AC magnetic field and fed with an $\mathrm{AC}$ transport current. The total $\mathrm{AC}$ loss can be separated in two contributions. First, the transport current loss influenced by an external AC magnetic field. The second contribution is the magnetisation loss that depends also on the transport current running through the conductor. In this paper the total $\mathrm{AC}$ loss is considered and the role of the electric and magnetic components is compared. This comparison is made with an available analytical model for the AC loss in an infinite slab and verified experimentally for a BSCCO/Ag tape conductor. For small transport currents the magnetisation loss dominates the total loss. When the current increases, a field dependent crossover occurs, after which the transport current loss also plays a role. Qualitatively the measurements can be described well in terms of the critical state model. For magnetic field parallel to the wide side of the conductor the CSM for an infinite slab describes the measurements also quantitatively.

\section{INTRODUCTION}

When a BSCCO/Ag tape is used in an application such as an electrical power cable or a (transformer) coil, the conductor is exposed to an external AC magnetic field and fed simultaneously with an AC transport current. On conductor level the total loss can be distinguished in a transport current loss part, energy delivered by the transport current power supply, and a magnetisation loss part, energy delivered by the external magnetic field. Depending on the amplitude of the alternating transport current and external magnetic field either the magnetisation loss or the transport current loss is the dominating term [1]. A set-up to measure the influence of an external alternating magnetic field on the transport current loss is already described [2] and the measurement results are published [2], [3]. This paper focuses on the magnetisation loss contribution to the total loss, especially in combination with an alternating transport current. The contribution of the two different loss contributions to the total AC loss is evaluated over a wide range of magnetic field and transport current

Manuscript received September 14, 1998

This research is supported by the Technology Foundation STW, applied science division of NWO and the technology programme of the Ministry of Economic Affairs. amplitudes. The total AC loss is compared with the available analytical model based on the critical state model for an infinitely large slab conductor [4]. For fully coupled filaments the thickness of the slab is related to the dimension of the filamentary zone. For a more accurate description a numerical model that can deal with the specific properties of the $\mathrm{BSCCO} / \mathrm{Ag}$ tape conductor (aspect ratio, $J_{c}(B)$ ) is necessary [1].

\section{THEORY}

Expressions for the total $\mathrm{AC}$ loss $Q_{t o t}$, i.e. the transport current loss $Q_{1}$ plus the magnetisation loss $Q_{m}$, are given in [4]. The model describes an 'infinite' slab fed with a transport current with amplitude $I$, in a parallel applied magnetic field with amplitude $B_{a}$ and a constant critical current density $J_{c}$. The loss for the normalised magnetic field, $\beta=B_{a} / B_{p}$, and the normalised transport current, $i=I_{t} / I_{c}$, is given by:

$$
\begin{aligned}
Q_{t o t}= & \frac{2 B_{p}^{2}}{3 \mu_{0}}\left(i^{3}+3 \beta^{2} i\right) \quad \text { for } \beta<i, \\
Q_{t o t}= & \frac{2 B_{p}^{2}}{3 \mu_{0}}\left(\beta^{3}+3 \beta i^{2}\right) \text { for } i<\beta<1, \\
Q_{t o t}= & \frac{2 B_{p}^{2}}{3 \mu_{0}}\left(\beta\left(3+i^{2}\right)-2\left(1-i^{3}\right)+6 i^{2} \frac{(1-i)^{2}}{(\beta-i)}\right. \\
& \left.-4 i^{2} \frac{(1-i)^{3}}{(\beta-i)^{2}}\right) \text { for } \beta>1,
\end{aligned}
$$

where $B_{p}=\mu_{\sigma} J_{c} a$ is the field of full penetration of a slab with thickness $2 a$. In the limit for $B_{a}>B_{p}$ this can be written as:

$Q_{t o t}=\frac{2 B_{p}^{2}}{3 \mu_{0}}\left(\beta\left(3+i^{2}\right)-2\left(1-i^{3}\right)\right)$ for $\beta>>1$.

For small values of the applied field (1) is dominated by:

$Q_{t r}=\frac{2 B_{p}^{2}}{3 \mu_{0}} i^{3}$ 
which is the transport current loss in a sheet for $B_{a}=0$. The $3 \beta^{2} i$ term in (1) represents the increase of the total loss due to an external alternating magnetic field. In (2)-(4) the magnetisation loss of a slab in parallel field can be recognised:

$$
\begin{array}{ll}
Q_{m}=\frac{2 B_{p}^{2}}{3 \mu_{0}} \beta^{3} & \text { for } \beta<1, \\
Q_{m}=\frac{2 B_{p}^{2}}{3 \mu_{0}}(3 \beta-2) & \text { for } \beta>1 .
\end{array}
$$

The total AC loss based on equations (1)-(3) is plotted in Fig. 1. The different regimes, $\beta<1, i<\beta<1$ and $\beta>1$ are indicated. Without transport current, the magnetisation loss is proportional to $\beta^{3}$ below the penetration field and above the penetration field with $\beta$. When an $\mathrm{AC}$ transport current is supplied simultaneously with an external alternating magnetic field, the total AC loss increases, especially in the regime with $\beta<i$. In the regime where $\beta>1$ the increase is relatively smaller. The penetration field for a typical $\mathrm{BSCCO} / \mathrm{Ag}$ tape with fully coupled filaments is around $10 \mathrm{mT}$ so, in most applications the situation $\beta>1$ will occur.

\section{MEASUREMENT SET-UP}

The magnetisation measurements are performed on a straight piece of $\mathrm{BSCCO} / \mathrm{Ag}$ sample of about $100 \mathrm{~mm}$ length. The sample is placed in a dipole magnet together with a set of

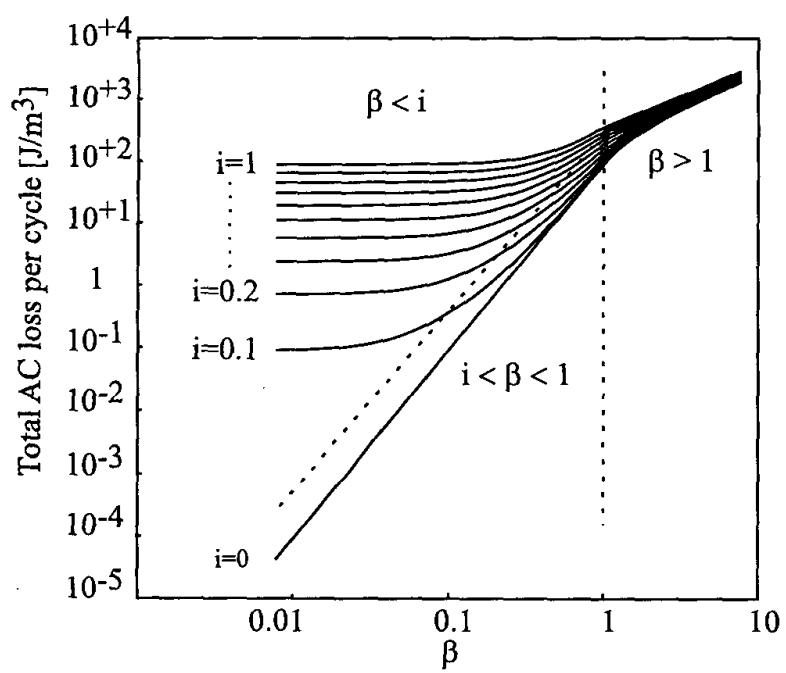

Fig.1. The total AC loss of a slab in parallel field for different magnetic field amplitudes and transport current amplitudes.

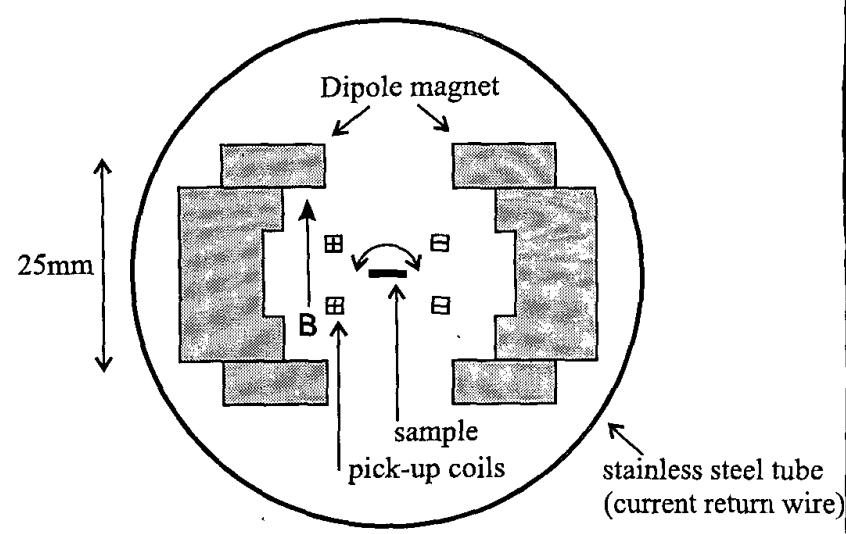

Fig. 2. Cross section of the magnetisation loss set-up (insert) with the dipole magnet, pick-up coils and the transport current return wire.

pick-up coils, see Fig. 2. A set of compensation coils, also placed in the magnet, is used to compensate the voltage induced by the magnetic field in the pick-up coils. A signal that remains when the two signals are subtracted is smaller than a few percent of the measured magnetisation loss in the entire experimental range. This signal is attributed to eddy currents in metallic parts of the insert, e.g. the dipole coil itself.

The sample is fed with an alternating transport current. The use of a stainless steel tube as current return wire for the sample current decouples the magnet current power supply and the transport current power supply [2], [3]. The transport current loss in an external alternating magnetic field, i.e. the electrical part of the total loss, is measured with a pair of voltage taps and a lock-in amplifier. Details of this set-up are described elsewhere [2], [3]. All measurements are performed at $77 \mathrm{~K}$ on a untwisted 85 filament $\mathrm{Bi}-2223$ sample with a pure silver matrix and a critical current density of about $130 \mathrm{~A} / \mathrm{mm}^{2}$.

\section{RESULTS}

The measured magnetisation loss of a $\mathrm{BSCCO} / \mathrm{Ag}$ tape exposed to an alternating magnetic field and fed simultaneously with an AC transport current is shown in Fig. 3. The loss is plotted in the form of the dimensionless loss function $\Gamma=Q \mu_{0} / 2 B_{a}^{2}$ for magnetic fields parallel and perpendicula to the wide side of the conductor. For the case without transpor current a field dependent loss function is determined with maximum around the field of full penetration. For paralle magnetic field smaller than $10 \mathrm{mT}$ the signal approaches the sensitivity limit of the measurement system. 


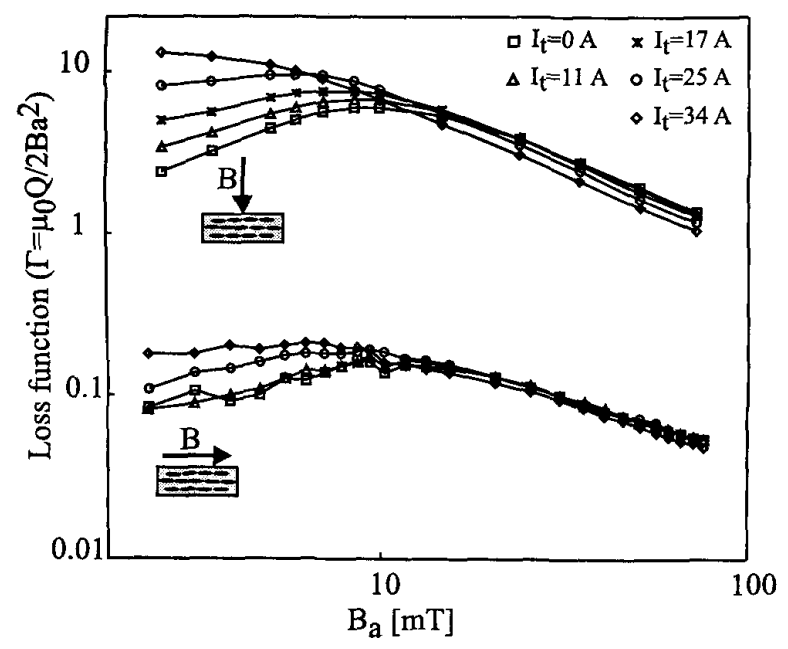

Fig. 3. The magnetisation loss in case of combined external field and alternating transport current, parallel and perpendicular field $(f=35 \mathrm{~Hz}$, $\mathrm{T}=77 \mathrm{~K}$ ). The lines are only a guide to the eye.

The magnetisation loss increases with increasing transport current when the magnetic field is smaller than $10 \mathrm{mT}$. For magnetic field amplitudes larger than $10 \mathrm{mT}$ the loss decreases, but this change is relatively small.

In the model for an 'infinite' slab behaving according to the critical state model, the magnetisation loss is not influenced by the transport current below the field of full penetration that depends on the transport current [5]. However in the measurements an increase of the magnetisation loss is observed. A similar effect is also observed in magnetisation experiments combined with a DC transport current [6]. The most probable explanation for this behaviour is the geometry of the BSCCO/Ag tape conductor that is not well described by the 'infinite' slab model. In a numerical simulation an increase of the magnetisation loss is observed when an additional alternating transport current is applied [1].

Above $B_{a} \approx 10 \mathrm{mT}$ the magnetisation loss decreases because the transport current occupies current space in the conductor which can not be used by screening currents. Because the transport current is an alternating current the decrease of the magnetisation loss is less than in case of a constant transport current. Also for alternating transport current amplitudes close to $I_{\mathrm{c}}\left(I_{t}=34 \mathrm{~A}\right)$ the decrease of the loss is hardly visible in Fig. 3. In case of a DC transport current the magnetisation loss would reduce to zero for $I_{i}=I_{c}$.

A comparison between the transport current loss influenced by an external alternating magnetic field and the magnetisation loss influenced by an alternating transport current is shown in Fig. 4 for magnetic field parallel to the tape conductor. The magnetisation loss remains almost constant when the transport

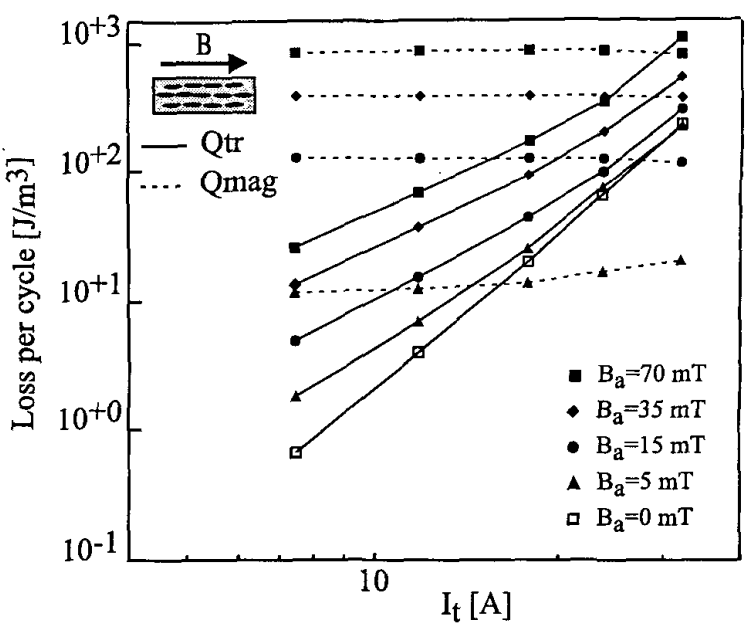

Fig. 4. The transport current loss in an external AC magnetic field and the magnetisation loss with an additional $\mathrm{AC}$ transport current in parallel field $(\mathrm{f}=35 \mathrm{~Hz}, \mathrm{~T}=77 \mathrm{~K}$ ). The lines are only a guide to the eye.

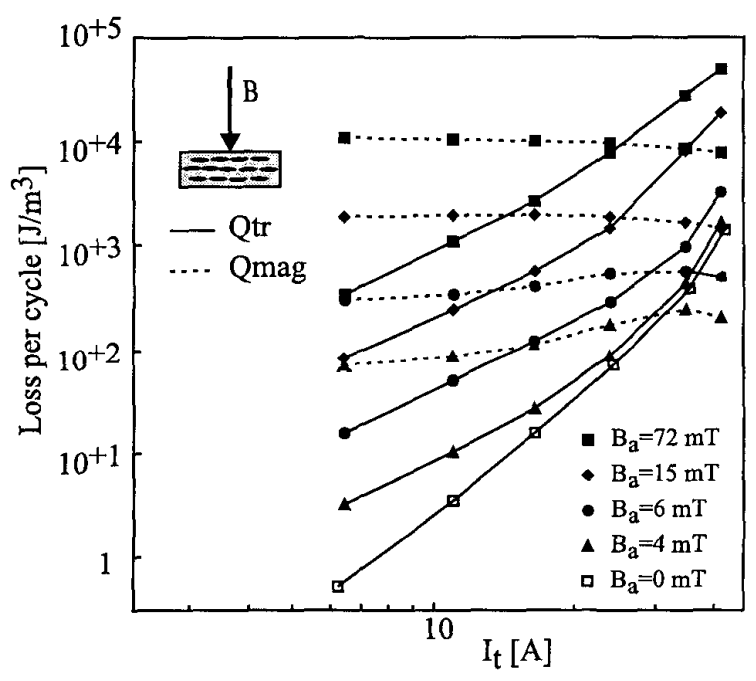

Fig. 5. The transport current loss in an external AC magnetic field and the magnetisation loss with additional $\mathrm{AC}$ transport current in perpendicular field $(f=35 \mathrm{~Hz}, \mathrm{~T}=77 \mathrm{~K}$ ). The lines are only a guide to the eye.

current amplitude increases. However, the transport current loss increases with the third power $\left(B_{a}=0 \mathrm{mT}\right)$ or second power of the current. At low transport current amplitudes the magnetisation loss is always dominant but when the current increases a crossover occurs after which the transport loss becomes dominant. The transport current amplitude at which the crossover takes place increases with increasing magnetic field amplitude. 
For a magnetic field perpendicular to the wide side of the tape the comparison between magnetisation loss and transport current loss is shown in Fig. 5. Qualitatively the picture is the same as for the parallel case. For a magnetic field amplitude of $4 \mathrm{mT}$, the magnetisation loss increases first with an increasing transport current amplitude but after reaching a maximum value it decreases. Also the decrease of the magnetisation loss for higher magnetic field amplitudes is more pronounced than in the case of parallel field. This is attributed to the much more pronounced reduction of $J_{c}$ due to the perpendicular applied magnetic field.

Finally the total $\mathrm{AC}$ loss, i.e. the magnetisation loss plus the transport current loss, is plotted for magnetic field parallel to the wide side of the tape conductor and compared with the slab model in Fig. 6. For $I_{t}=0$ the total AC loss is equal to the magnetisation loss. When an additional alternating transport current is supplied the total AC loss increases. Above $B_{a} \approx 20 \mathrm{mT}$ the increase is much less than for a low magnetic field. The increase at low magnetic field amplitudes is much larger because the crossover between magnetic loss and transport current loss (Fig. 4) takes place at lower transport current amplitudes. Above $B_{a}=10 \mathrm{mT}$ the total $\mathrm{AC}$ loss, for constant $I_{t}$, increases almost linear with the magnetic field amplitude. The measurements are also compared with the slab model. In the case with a small transport current $\left(I_{t} \ll I_{c}\right)$ a rather good agreement is observed. For the case of $I_{t}=I_{c}(=34 \mathrm{~A})$ a substantial difference is observed. The major part of this difference is attributed to the fact that the calculation is based on the critical state model for a homogeneous 'infinite' slab. The $\mathrm{BSCCO} / \mathrm{Ag}$ tape conductor has a far from constant $J_{c}(B)$, and a 2-dimensional geometry. A more detailed analysis has to be based on numerical calculations as in [1].

\section{CONCLUSIONS}

The magnetisation loss of an BSCCO/Ag tape conductor exposed to an alternating magnetic field and fed simultaneously with an alternating transport current is measured. Below the penetration field the magnetisation loss increases and above the penetration field the magnetisation loss decreases due to the alternating transport current. The influence of an transport current is larger for magnetic field perpendicular to the wide side of the tape than for magnetic field parallel to the wide side of the tape. The magnetisation loss is compared with the transport current loss for a wide range of magnetic field and transport current amplitudes. For small transport currents the magnetisation loss is dominant. When the transport current amplitude increases a crossover occurs after which the transport current loss becomes dominant. The transport current amplitude at which the

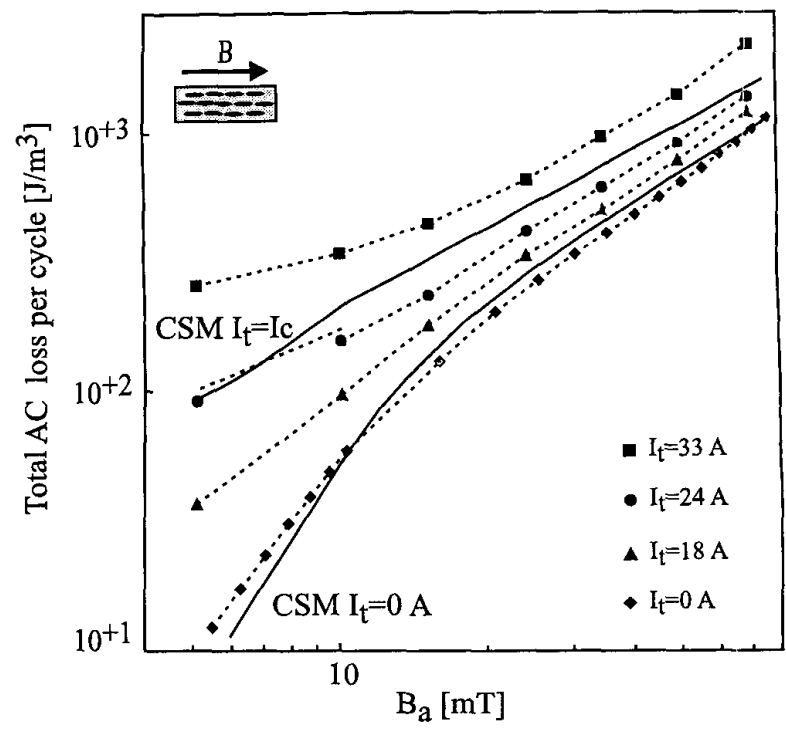

Fig. 6. The total AC loss in parallel field $(\mathrm{f}=35 \mathrm{~Hz}, \mathrm{~T}=77 \mathrm{~K}$ ). The dashed lines are only guides to the eye. The dots are measured points. The solid lines represents the CSM model according to Eq. (1)-(3).

crossover takes place is field dependent and increases for a parallel applied magnetic field.

The behaviour of the $\mathrm{BSCCO} / \mathrm{Ag}$ tape in the situation of combined alternating magnetic field and transport current can be described qualitatively with the critical state model, both for magnetic field parallel and perpendicular to the wide side of the conductor. The critical state model applied to a slab in parallel magnetic field describes the total AC loss for magnetic field parallel to the tape conductor also quantitatively very well.

\section{REFERENCES}

[1] T. Yazawa, J. J. Rabbers, B. ten Haken, H. H. J. ten Kate and Y. Yamada, 'Numerical calculation of current density distributions in HTS tapes with finite thickness in self field and external field', Physica $C 310$ pp. 36-41, 1998.

[2] J. J. Rabbers, B. ten Haken, H. H. J. ten Kate, 'Measuring transport current loss of $\mathrm{BSCCO} / \mathrm{Ag}$ tapes exposed to external $\mathrm{AC}$ magnetic field', Physica C 310, pp. 101-105, 1998.

[3] J. J. Rabbers, B. ten Haken, F. Gömöry, H. H. J. ten Kate, 'Self-field loss of BSCCO/Ag tape in external AC magnetic field', Physica C 300, Pp. 1-5, 1998.

[4] W. J. Carr Jr., 'AC loss from the combined action of transport current and applied field', IEEE Transactions on Magnetics, 15(1) pp. 240 243,1979 .

[5] M. N. Wilson, 'Superconducting magnets', Clarendon Press, Oxford 1983.

[6] M. Ciszek et al. 'AC magnetic losses in multifilamentary $A g / B i-2223$ tape carrying DC transport current",Inst. Phys. Conf. Ser. No 158, paper presented at Eucas 1997 Veldhoven The Netherlands pp. 1425-1428. 